\title{
Rh(III)-catalyzed C-H activation of benzamides: Coupling with quinones
}

\author{
Zisong Qi, Xingwei Li* \\ Dalian Institute of Chemical Physics, Chinese Academy of Sciences, Dalian 116023, Liaoning, China
}

\section{A R T I C L E I N F O}

Article history:

Received 25 September 2014

Accepted 10 November 2014

Published 20 January 2015

Keywords:

Rhodium(III)

$\mathrm{C}-\mathrm{H}$ activation

Benzamides

Arylquinones
A B S T R A C T

$\mathrm{Rh}$ (III)-catalyzed $\mathrm{C}-\mathrm{H}$ activation of $\mathrm{N}$-(alkyl)benzamides in the oxidative coupling with various quinones. In addition, under redox-neutral conditions, 2-hydroxy-6H-benzo[c]chromen-6-ones were also obtained via a cascade of cross-coupling followed by lactonization.

(C) 2015, Dalian Institute of Chemical Physics, Chinese Academy of Sciences. Published by Elsevier B.V. All rights reserved.

\section{Introduction}

Transition-metal-catalyzed direct functionalization of $\mathrm{C}-\mathrm{H}$ bonds has become an increasingly important strategy for the construction of complex organic molecules [1-3]. Palladium, ruthenium, iridium, and copper catalysts are particularly well known to serve this purpose [4-19]. Although much progress has been made, the development of new synthetic methods is still necessary, especially via a $\mathrm{C}-\mathrm{H}$ activation pathway. $\mathrm{Rh}^{\mathrm{III}} \mathrm{Cp}$ * represent one of the most frequently used catalysts that enabled a vast number of transformations through coupling of $\mathrm{C}-\mathrm{H}$ bonds with alkenes along with other molecules [20-57]. However, the olefins are mostly limited to acrylates and styrenes. There are only a few reports on the applications of quinones as an olefin partner under oxidative conditions [58]. The rarity is probably due to the difficulty of $\beta$-hydride elimination following syn migratory insertion of a $\mathrm{Rh}-\mathrm{C}$ bond into the $\mathrm{C}=\mathrm{C}$ bond of a quinone. In addition, the steric effect of this special disubstituted olefin may also be accountable [59-63].
On the other hand, quinones represent an important class of biologically active molecules that are widely distributed in natural products [64-67]. Both natural and synthetic medicinal agents have been developed that are based on the quinone structure due to their chemotherapeutic value such as antitumor, antifungal, and antibacterial [68-72]. The quinone moiety is also involved in many bioenergetic processes and plays a vital role in electron-transport processes [73]. Aryl-substituted quinones are very useful in photosynthesis and the dye industry owing to their unique visual and electronic properties $[74,75]$. Thus, developing powerful synthetic methods of arylquinones is very necessary to fulfill the practical requirements. Arylation of quinones has been reported by some groups [76-90]. However, these approaches often suffer from poor atom-economy, substrate limitations, stoichiometric amounts of metals or high temperature. Although much progress has been made, the development of new synthetic methods is still necessary, especially via a $\mathrm{C}-\mathrm{H}$ activation pathway. Herein we report $\mathrm{Rh}(\mathrm{III})$-catalyzed $\mathrm{C}-\mathrm{H}$ activation of

\footnotetext{
* Corresponding author. Tel: +86-411-84379089; E-mail: xwli@dicp.ac.cn This work was supported by the Dalian Institute of Chemical Physics, Chinese Academy of Sciences and the National Natural Science Foundation of China (21272231). 
$N$-(alkyl)benzamides in the coupling with quinones, leading to synthesis of arylated quinones.

\section{Experimental}

\subsection{General}

All reactions were carried out using Schlenk techniques or in a nitrogen-filled glovebox. NMR spectra were recorded on a Bruker $400 \mathrm{MHz}$ NMR spectrometer in the solvents indicated. The chemical shift is given in dimensionless $\delta$ values and is frequency referenced relative to TMS in ${ }^{1} \mathrm{H}$ and ${ }^{13} \mathrm{C}$ NMR spectroscopy. HRMS data were obtained on an Agilent 6540 Q-Tof. Column chromatography was performed on silica gel (300-400 mesh) using ethyl acetate (EA)/petroleum ether (PE). All chemicals were obtained from commercial sources and were used as received unless otherwise noted.

\subsection{Preparation of compounds}

\subsubsection{General procedure for the synthesis of compounds $\mathbf{3}$}

$N$-(alkyl)benzamides (0.2 mmol), quinones $(0.3 \mathrm{mmol})$, [RhCp* $\left.\mathrm{Cl}_{2}\right]_{2}$ (4 mol\%), $\mathrm{AgSbF}_{6}(16 \mathrm{~mol} \%), \mathrm{Ag}_{2} \mathrm{CO}_{3}$ (2.0 equiv) and dioxane $(3 \mathrm{~mL})$ were charged into the sealed tube. The reaction mixture was stirred at $100{ }^{\circ} \mathrm{C}$ for $12 \mathrm{~h}$. After cooled to room temperature, the solvent was removed under reduced pressure and the residue was purified by silica gel chromatography using PE/EA to afford compounds 3.

\subsubsection{General procedure for the synthesis of compounds 4}

$\mathrm{N}$-(tert-butyl)benzamides $(0.2 \mathrm{mmol})$, quinones $(0.3 \mathrm{mmol})$, $\left[\mathrm{RhCp}^{*} \mathrm{Cl}_{2}\right]_{2}$ (4 mol\%), $\mathrm{AgSbF}_{6}$ (30 mol\%), AgOAc (0.2 equiv) and acetone $(4 \mathrm{~mL})$ were charged into the sealed tube. The reaction mixture was stirred at $100{ }^{\circ} \mathrm{C}$ for $12 \mathrm{~h}$. After cooled to room temperature, the solvent was removed under reduced pressure and the residue was purified by silica gel chromatography using etroleum ether/ethyl acetate to afford compounds 4.

\subsection{Spectral data for products}

$N$-(tert-butyl)-2',5'-dioxo-2',5'-dihydro-[1,1'-biphenyl]-2carboxamide (3a). ${ }^{1} \mathrm{H}$ NMR (400 MHz, $\mathrm{CDCl}_{3}$ ) $\delta 7.53$ (dd, $J=7.4$, $1.5 \mathrm{~Hz}, 1 \mathrm{H}), 7.48(\mathrm{dd}, J=7.4,1.6 \mathrm{~Hz}, 1 \mathrm{H}), 7.44(\mathrm{td}, J=7.5,1.5 \mathrm{~Hz}$, $1 \mathrm{H}), 7.29(\mathrm{dd}, J=7.4,1.2 \mathrm{~Hz}, 1 \mathrm{H}), 6.80(\mathrm{~d}, J=1.1 \mathrm{~Hz}, 2 \mathrm{H}), 6.75-$ $6.72(\mathrm{~m}, 1 \mathrm{H}), 6.01(\mathrm{~s}, 1 \mathrm{H}), 1.38(\mathrm{~s}, 9 \mathrm{H}) .{ }^{13} \mathrm{C}$ NMR $(100 \mathrm{MHz}$, $\left.\mathrm{CDCl}_{3}\right) \delta 187.6,185.8,168.4,149.5,137.6,137.03,136.97$, 133.2, 131.3, 130.9, 130.4, 129.8, 126.5, 52.0, 28.7. HRMS: [M + $\mathrm{H}]^{+}$calculated for $\mathrm{C}_{7} \mathrm{H}_{18} \mathrm{NO}_{3}: 284.1287$, found 284.1290 .

$N$-(tert-butyl)-5-methyl-2',5'-dioxo-2',5'-dihydro-[1,1'biphenyl]-2-carboxamide (3b). ${ }^{1} \mathrm{H}$ NMR (400 $\left.\mathrm{MHz} \mathrm{CDCl}_{3}\right) \delta$ $7.42(\mathrm{~d}, J=7.8 \mathrm{~Hz}, 1 \mathrm{H}), 7.24(\mathrm{~d}, J=7.8 \mathrm{~Hz}, 1 \mathrm{H}), 7.09$ (s, 1H), 6.80 $(\mathrm{s}, 2 \mathrm{H}), 6.72(\mathrm{~s}, 1 \mathrm{H}), 5.96(\mathrm{~s}, 1 \mathrm{H}), 2.39(\mathrm{~s}, 3 \mathrm{H}), 1.37(\mathrm{~s}, 9 \mathrm{H}) .{ }^{13} \mathrm{C}$ NMR (100 MHz, $\left.\mathrm{CDCl}_{3}\right) \delta 187.6,185.9,168.4,149.8,141.3$, 137.1, 137.0, 134.9, 133.3, 131.2, 131.0, 130.3, 126.5, 51.9, 28.8, 21.4. HRMS: $[\mathrm{M}+\mathrm{H}]^{+}$calculated for $\mathrm{C}_{18} \mathrm{H}_{20} \mathrm{NO}_{3}$ : 298.1443, found 298.1440 .
$N$-(tert-butyl)-5-methoxy-2',5'-dioxo-2',5'-dihydro-[1,1'biphenyl]-2-carboxamide (3c). ${ }^{1} \mathrm{H}$ NMR (400 $\left.\mathrm{MHz} \mathrm{CDCl}_{3}\right) \delta$ $7.46(\mathrm{~d}, J=8.5 \mathrm{~Hz}, 1 \mathrm{H}), 6.92(\mathrm{dd}, J=8.5,2.6 \mathrm{~Hz}, 1 \mathrm{H}), 6.80(\mathrm{dd}, J=$ 4.1, $2.2 \mathrm{~Hz}, 3 \mathrm{H}$ ), 6.72 (d, $J=1.5 \mathrm{~Hz}, 1 \mathrm{H}), 5.93$ (s, 1H), 3.84 (s, 3H), $1.37(\mathrm{~s}, 9 \mathrm{H}) .{ }^{13} \mathrm{C}$ NMR $\left(100 \mathrm{MHz}, \mathrm{CDCl}_{3}\right) \delta 187.6,185.7$, $168.1,161.4,149.8,137.2,136.9,135.2,131.0,130.0,128.1$, 116.3, 114.4, 55.7, 51.9, 28.8. HRMS: $[\mathrm{M}+\mathrm{H}]^{+}$calculated for $\mathrm{C}_{18} \mathrm{H}_{20} \mathrm{NO}_{4}: 314.1392$, found 314.1391 .

$N$-(tert-butyl)-5-fluoro-2',5'-dioxo-2',5'-dihydro-[1,1'biphenyl]-2-carboxamide (3d). ${ }^{1} \mathrm{H}$ NMR (400 $\left.\mathrm{MHz} \mathrm{CDCl}_{3}\right) \delta$ $7.53(\mathrm{dd}, J=8.5,5.4 \mathrm{~Hz}, 1 \mathrm{H}), 7.13(\mathrm{td}, J=8.3,2.6 \mathrm{~Hz}, 1 \mathrm{H}), 7.01$ (dd, $J=8.8,2.6 \mathrm{~Hz}, 1 \mathrm{H}), 6.82(\mathrm{~d}, J=1.0 \mathrm{~Hz}, 2 \mathrm{H}), 6.73(\mathrm{~s}, 1 \mathrm{H}), 5.94$ (s, 1H), 1.37 (s, 9H). ${ }^{13} \mathrm{C}$ NMR (100 MHz, CDCl 3 ) $\delta 187.3,185.4$, 167.5, 163.7 (d, $J=250.9 \mathrm{~Hz}), 148.3$ (d, $J=1.5 \mathrm{~Hz}), 137.1,137.0$, 135.7 (d, $J=8.2 \mathrm{~Hz}), 134.0$ (d, $J=3.4 \mathrm{~Hz}), 131.7,128.6$ (d, $J=8.7$ Hz), 117.7 (d, $J=22.9 \mathrm{~Hz}$ ), 116.3 (d, $J=21.1 \mathrm{~Hz}$ ), 52.2, 28.7. HRMS: $[\mathrm{M}+\mathrm{H}]^{+}$calculated for $\mathrm{C}_{17} \mathrm{H}_{17} \mathrm{FNO}_{3}$ : 302.1192 , found 302.1181.

$N$-(tert-butyl)-5-chloro-2',5'-dioxo-2',5'-dihydro-[1,1'biphenyl]-2-carboxamide (3e). ${ }^{1} \mathrm{H}$ NMR $\left(400 \mathrm{MHz}, \mathrm{CDCl}_{3}\right) \delta$ 7.47 (d, $J=8.2 \mathrm{~Hz}, 1 \mathrm{H}), 7.41$ (dd, $J=8.2,2.0 \mathrm{~Hz}, 1 \mathrm{H}), 7.28$ (d, $J=$ $2.1 \mathrm{~Hz}, 1 \mathrm{H}), 6.81(\mathrm{~s}, 2 \mathrm{H}), 6.74(\mathrm{~s}, 1 \mathrm{H}), 5.97(\mathrm{~s}, 1 \mathrm{H}), 1.37(\mathrm{~s}, 9 \mathrm{H})$. ${ }^{13} \mathrm{C}$ NMR $\left(100 \mathrm{MHz}, \mathrm{CDCl}_{3}\right) \delta 187.2,185.4,167.5,148.1,137.1$, 137.0, 136.8, 136.1, 134.9, 131.7, 130.4, 129.7, 127.9, 52.2, 28.7. HRMS: $[\mathrm{M}+\mathrm{H}]^{+}$calculated for $\mathrm{C}_{17} \mathrm{H}_{17} \mathrm{ClNO}_{3}$ : 318.0897 , found 318.0898.

5-Bromo-N-(tert-butyl)-2',5'-dioxo-2',5'-dihydro-[1,1'biphenyl]-2-carboxamide (3f). ${ }^{1} \mathrm{H}$ NMR $\left(400 \mathrm{MHz} \mathrm{CDCl}_{3}\right) \delta$ $7.58(\mathrm{dd}, J=8.2,2.0 \mathrm{~Hz}, 1 \mathrm{H}), 7.44(\mathrm{~d}, J=1.9 \mathrm{~Hz}, 1 \mathrm{H}), 7.39$ (d, $J=$ $8.2 \mathrm{~Hz}, 1 \mathrm{H}), 6.81(\mathrm{~s}, 2 \mathrm{H}), 6.74(\mathrm{~s}, 1 \mathrm{H}), 5.95(\mathrm{~s}, 1 \mathrm{H}), 1.37(\mathrm{~s}, 9 \mathrm{H})$. ${ }^{13} \mathrm{C}$ NMR $\left(100 \mathrm{MHz} \mathrm{CDCl}_{3}\right) \delta 187.2,185.4,167.6,148.0,137.1$, 137.0, 136.5, 135.0, 133.3, 132.7, 131.8, 128.0, 124.9, 52.3, 28.7. HRMS: $[\mathrm{M}+\mathrm{H}]^{+}$calculated for $\mathrm{C}_{17} \mathrm{H}_{17} \mathrm{BrNO}_{3}$ : 362.0392 , found 362.0391.

$N$-(tert-butyl)-2"',5"'-dioxo-2',5'-dihydro-[1,1':3',1'"terphenyl]-4'-carboxamide (3g). ${ }^{1} \mathrm{H}$ NMR (400 MHz, $\left.\mathrm{CDCl}_{3}\right) \delta$ 7.65 (dd, $J=7.9,1.6 \mathrm{~Hz}, 1 \mathrm{H}), 7.61-7.54(\mathrm{~m}, 3 \mathrm{H}), 7.49-7.43(\mathrm{~m}$, $3 \mathrm{H}), 7.39(\mathrm{t}, J=7.2 \mathrm{~Hz}, 1 \mathrm{H}), 6.82(\mathrm{~s}, 2 \mathrm{H}), 6.80(\mathrm{~d}, J=1.3 \mathrm{~Hz}, 1 \mathrm{H})$, $6.04(\mathrm{~s}, 1 \mathrm{H}), 1.40(\mathrm{~s}, 9 \mathrm{H}) .{ }^{13} \mathrm{C}$ NMR $\left(100 \mathrm{MHz}, \mathrm{CDCl}_{3}\right) \delta 187.6$, 185.9, 168.2, 149.6, 144.0, 139.5, 137.1, 137.1, 136.3, 133.8, $131.4,129.3,129.2,128.4,128.3,127.3,127.1,52.1,28.8$. HRMS: $[\mathrm{M}+\mathrm{H}]^{+}$calculated for $\mathrm{C}_{23} \mathrm{H}_{22} \mathrm{NO}_{3}$ : 360.1600 , found 360.1600 .

$N$-(tert-butyl)-2',5'-dioxo-5-(trifluoromethyl)-2',5'-dihydro[1,1'-biphenyl]-2-carboxamide (3h). ${ }^{1} \mathrm{H}$ NMR (400 MHz, $\left.\mathrm{CDCl}_{3}\right)$ $\delta 7.72$ (dd, $J=8.0,1.0 \mathrm{~Hz}, 1 \mathrm{H}), 7.64(\mathrm{~d}, J=8.0 \mathrm{~Hz}, 1 \mathrm{H}), 7.55(\mathrm{~s}$, $1 \mathrm{H}), 6.84(\mathrm{~d}, J=2.2 \mathrm{~Hz}, 2 \mathrm{H}), 6.80(\mathrm{~d}, J=2.1 \mathrm{~Hz}, 1 \mathrm{H}), 5.97(\mathrm{~s}, 1 \mathrm{H})$, 1.39 (s, 9H). ${ }^{13} \mathrm{C}$ NMR (100 MHz, $\left.\mathrm{CDCl}_{3}\right) \delta 187.1,185.4,167.3$, $147.9,140.9,137.3,136.9,134.0,132.9$ (q, $J=33.2 \mathrm{~Hz}$ ), 132.2, $127.4(\mathrm{q}, J=3.6 \mathrm{~Hz}), 127.1,126.7(\mathrm{q}, J=3.7 \mathrm{~Hz}), 123.4(\mathrm{q}, J=$ $267.7 \mathrm{~Hz}), 52.5$, 28.7. HRMS: $[\mathrm{M}+\mathrm{H}]^{+}$calculated for $\mathrm{C}_{18} \mathrm{H}_{17} \mathrm{~F}_{3} \mathrm{NO}_{3}$ : 352.1161, found 352.1157.

Methyl 6-(tert-butylcarbamoyl)-2',5'-dioxo-2',5'-dihydro-[1,1'biphenyl]-3-carboxylate (3i). ${ }^{1} \mathrm{H}$ NMR (400 MHz, $\left.\mathrm{CDCl}_{3}\right) \delta 8.10$ (dd, $J=8.0,1.4 \mathrm{~Hz}, 1 \mathrm{H}), 7.96(\mathrm{~d}, J=1.2 \mathrm{~Hz}, 1 \mathrm{H}), 7.59$ (d, $J=8.0$ $\mathrm{Hz}, 1 \mathrm{H}), 6.81$ (d, $J=6.4 \mathrm{~Hz}, 3 \mathrm{H}), 6.04$ (s, 1H), 3.94 (s, 3H), 1.39 (s, 9H). ${ }^{13} \mathrm{C}$ NMR $\left(100 \mathrm{MHz}, \mathrm{CDCl}_{3}\right) \delta 187.3,185.6,167.7,165.8$, 
$148.4,141.5,137.2,136.9,133.4,132.3,132.0,131.5,131.0$, 126.7, 52.7, 52.4, 28.7. HRMS: $[\mathrm{M}+\mathrm{H}]^{+}$calculated for $\mathrm{C}_{19} \mathrm{H}_{20} \mathrm{NO}_{5}: 342.1341$, found 342.1340.

$N$-(tert-butyl)-4-methyl-2',5'-dioxo-2',5'-dihydro-[1,1'biphenyl]-2-carboxamide (3j). ${ }^{1} \mathrm{H}$ NMR (400 $\left.\mathrm{MHz}^{\mathrm{CDCl}} 3\right) \delta$ 7.33 (s, 1H), 7.29 (d, $J=8.6 \mathrm{~Hz}, 1 \mathrm{H}$ ), 7.17 (d, $J=7.7 \mathrm{~Hz}, 1 \mathrm{H}), 6.78$ $(\mathrm{s}, 2 \mathrm{H}), 6.71(\mathrm{~s}, 1 \mathrm{H}), 6.00(\mathrm{~s}, 1 \mathrm{H}), 2.41(\mathrm{~s}, 3 \mathrm{H}), 1.38(\mathrm{~s}, 9 \mathrm{H}) .{ }^{13} \mathrm{C}$ NMR $\left(100 \mathrm{MHz}, \mathrm{CDCl}_{3}\right) \delta 187.6,186.0,168.5,149.4,140.2$, 137.6, 137.0, 136.9, 131.4, 131.0, 130.4, 130.2, 127.3, 51.9, 28.7, 21.3. HRMS: $[\mathrm{M}+\mathrm{H}]^{+}$calculated for $\mathrm{C}_{18} \mathrm{H}_{20} \mathrm{NO}_{3}$ : 298.1443, found 298.1446.

$N$-(tert-butyl)-4-methoxy-2',5'-dioxo-2',5'-dihydro-[1,1'biphenyl]-2-carboxamide (3k). ${ }^{1} \mathrm{H}$ NMR (400 $\left.\mathrm{MHz} \mathrm{CDCl}_{3}\right) \delta$ $7.23(\mathrm{~d}, J=8.4 \mathrm{~Hz}, 1 \mathrm{H}), 7.05(\mathrm{~d}, J=2.5 \mathrm{~Hz}, 1 \mathrm{H}), 7.00$ (dd, $J=8.4$, $2.6 \mathrm{~Hz}, 1 \mathrm{H}), 6.79$ (d, $J=1.1 \mathrm{~Hz}, 2 \mathrm{H}), 6.72(\mathrm{~d}, J=1.1 \mathrm{~Hz}, 1 \mathrm{H}), 5.91$ (s, 1H), 3.87 (s, 3H), 1.37 (s, 9H). $\left.{ }^{13} \mathrm{C} \mathrm{NMR} \mathrm{(100} \mathrm{MHz,} \mathrm{CDCl}_{3}\right) \delta$ 187.7, 186.2, 168.2, 160.9, 149.0, 139.2, 137.1, 137.0, 131.9, $130.9,125.3,115.1,113.6,55.9,52.1,28.8$. HRMS: $[\mathrm{M}+\mathrm{H}]^{+}$calculated for $\mathrm{C}_{18} \mathrm{H}_{20} \mathrm{NO}_{4}$ : 314.1392, found 314.1394.

$N$-(tert-butyl)-6-methoxy-2',5'-dioxo-2',5'-dihydro-[1,1'biphenyl]-2-carboxamide (3k'). ${ }^{1} \mathrm{H}$ NMR (400 $\left.\mathrm{MHz} \mathrm{CDCl}_{3}\right) \delta$ $7.38(\mathrm{t}, J=8.0 \mathrm{~Hz}, 1 \mathrm{H}), 7.11(\mathrm{~d}, J=7.7 \mathrm{~Hz}, 1 \mathrm{H}), 7.02(\mathrm{~d}, J=8.4 \mathrm{~Hz}$, $1 \mathrm{H}), 6.84(\mathrm{~d}, J=10.1 \mathrm{~Hz}, 1 \mathrm{H}), 6.80$ (dd, $J=10.1,2.4 \mathrm{~Hz}, 1 \mathrm{H}), 6.74$ (d, $J=2.4 \mathrm{~Hz}, 1 \mathrm{H}), 5.70$ (s, 1H), 3.77 (s, 3H), 1.32 (s, 9H). ${ }^{13} \mathrm{C}$ NMR $\left(100 \mathrm{MHz}, \mathrm{CDCl}_{3}\right) \delta 187.5,186.3,168.1,157.4,144.8$, 139.2, 137.1, 136.8, 134.4, 130.6, 121.0, 118.9, 113.0, 56.2, 52.0, 28.7. HRMS: $[\mathrm{M}+\mathrm{H}]^{+}$calculated for $\mathrm{C}_{18} \mathrm{H}_{20} \mathrm{NO}_{4}$ : 314.1392 , found 314.1395 .

$N$-(tert-butyl)-4-chloro-2',5'-dioxo-2',5'-dihydro-[1,1'biphenyl]-2-carboxamide (3l). ${ }^{1} \mathrm{H}$ NMR (400 $\left.\mathrm{MHz}^{\mathrm{CDCl}} 3\right) \delta$ $7.50(\mathrm{~d}, J=2.0 \mathrm{~Hz}, 1 \mathrm{H}), 7.47(\mathrm{dd}, J=8.1,2.1 \mathrm{~Hz}, 1 \mathrm{H}), 7.23$ (d, $J=$ $8.1 \mathrm{~Hz}, 1 \mathrm{H}), 6.81(\mathrm{~s}, 2 \mathrm{H}), 6.74(\mathrm{~s}, 1 \mathrm{H}), 5.96(\mathrm{~s}, 1 \mathrm{H}), 1.38(\mathrm{~s}, 9 \mathrm{H})$. ${ }^{13} \mathrm{C}$ NMR $\left(100 \mathrm{MHz}, \mathrm{CDCl}_{3}\right) \delta 187.3,185.6,167.2,148.2,139.2$, 137.1, 137.0, 135.9, 131.7, 131.7, 131.5, 130.9, 127.0, 52.4, 28.7. HRMS: $[\mathrm{M}+\mathrm{H}]^{+}$calculated for $\mathrm{C}_{17} \mathrm{H}_{17} \mathrm{ClNO}_{3}$ : 318.0897, found 318.0897.

$N$-(tert-butyl)-6-chloro-2',5'-dioxo-2',5'-dihydro-[1,1'biphenyl]-2-carboxamide (3l'). ${ }^{1} \mathrm{H}$ NMR (400 $\left.\mathrm{MHz}^{\mathrm{CDCl}} 3\right) \delta$ $7.53(\mathrm{~d}, J=7.8 \mathrm{~Hz}, 1 \mathrm{H}), 7.44-7.35(\mathrm{~m}, 2 \mathrm{H}), 6.90-6.82(\mathrm{~m}, 2 \mathrm{H})$, $6.74(\mathrm{~d}, J=2.0 \mathrm{~Hz}, 1 \mathrm{H}), 5.68(\mathrm{~s}, 1 \mathrm{H}), 1.31(\mathrm{~s}, 9 \mathrm{H}) .{ }^{13} \mathrm{C}$ NMR $(100$ $\mathrm{MHz}_{\mathrm{CDCl}}$ ) $\delta 187.0,185.7,167.1,145.5,139.7,137.1,136.8$, 134.7, 134.3, 131.5, 130.9, 130.4, 125.1, 52.3, 28.7. HRMS: [M + $\mathrm{H}]^{+}$calculated for $\mathrm{C}_{17} \mathrm{H}_{17} \mathrm{ClNO}_{3}: 318.0897$, found 318.0899.

$N$-(tert-butyl)-6-fluoro-2',5'-dioxo-2',5'-dihydro-[1,1'biphenyl]-2-carboxamide (3m). ${ }^{1} \mathrm{H}$ NMR (400 MHz, $\left.\mathrm{CDCl}_{3}\right) \delta$ 7.42 (td, $J=8.0,5.3 \mathrm{~Hz}, 1 \mathrm{H}), 7.33(\mathrm{~d}, J=7.2 \mathrm{~Hz}, 1 \mathrm{H}), 7.24(\mathrm{t}, J=$ $8.8 \mathrm{~Hz}, 1 \mathrm{H}), 6.87-6.79(\mathrm{~m}, 3 \mathrm{H}), 5.89(\mathrm{~s}, 1 \mathrm{H}), 1.36(\mathrm{~s}, 9 \mathrm{H}) .{ }^{13} \mathrm{C}$ NMR (100 MHz, $\left.\mathrm{CDCl}_{3}\right) \delta 187.1,185.4,167.2(\mathrm{~d}, J=2.7 \mathrm{~Hz})$, 160.1 (d, $J=248.7 \mathrm{~Hz}$ ), 141.8, 139.7 (d, $J=1.7 \mathrm{~Hz}$ ), 137.1, 137.0, $134.4(\mathrm{~d}, J=3.0 \mathrm{~Hz}), 131.0$ (d, $J=8.7 \mathrm{~Hz}), 122.4$ (d, $J=3.4 \mathrm{~Hz}$ ), 120.2 (d, $J=15.7 \mathrm{~Hz}$ ), 118.2 (d, $J=22.9 \mathrm{~Hz}$ ), 52.2, 28.7. HRMS: $[\mathrm{M}+\mathrm{H}]^{+}$calculated for $\mathrm{C}_{17} \mathrm{H}_{17} \mathrm{FNO}_{3}:$ 302.1192, found 302.1196.

$N$-(tert-butyl)-3-methoxy-2',5'-dioxo-2',5'-dihydro-[1,1'biphenyl]-2-carboxamide (3n). ${ }^{1} \mathrm{H}$ NMR (400 $\left.\mathrm{MHz} \mathrm{CDCl}_{3}\right) \delta$ 7.45-7.37 (m, 1H), $7.05(\mathrm{~d}, J=8.4 \mathrm{~Hz}, 1 \mathrm{H}), 6.96(\mathrm{~s}, 1 \mathrm{H}), 6.89$ (d, $J$ $=7.6 \mathrm{~Hz}, 1 \mathrm{H}), 6.81-6.78(\mathrm{~m}, 2 \mathrm{H}), 6.67(\mathrm{~d}, J=2.1 \mathrm{~Hz}, 1 \mathrm{H}), 3.92(\mathrm{~s}$, $3 \mathrm{H}), 1.36(\mathrm{~s}, 9 \mathrm{H}) .{ }^{13} \mathrm{C}$ NMR $\left(100 \mathrm{MHz}, \mathrm{CDCl}_{3}\right) \delta 187.8,185.5$,
165.0, 156.5, 150.9, 137.2, 136.9, 136.1, 131.4, 129.8, 125.2, 123.3, 113.4, 56.5, 51.7, 28.9. HRMS: $[\mathrm{M}+\mathrm{H}]^{+}$calculated for $\mathrm{C}_{18} \mathrm{H}_{20} \mathrm{NO}_{4}: 314.1392$, found 314.1398.

$N$-(tert-butyl)-3-fluoro-2',5'-dioxo-2',5'-dihydro-[1,1'biphenyl]-2-carboxamide (3o). ${ }^{1} \mathrm{H}$ NMR (400 $\left.\mathrm{MHz}, \mathrm{CDCl}_{3}\right) \delta$ 7.48-7.41 (m, 1H), 7.20 (ddd, $J=10.2,8.4,0.9 \mathrm{~Hz}, 1 \mathrm{H}$ ), 7.09 (dd, $J=7.6,0.7 \mathrm{~Hz}, 1 \mathrm{H}), 6.81(\mathrm{~d}, J=1.2 \mathrm{~Hz}, 2 \mathrm{H}), 6.73(\mathrm{t}, J=1.1 \mathrm{~Hz}$, 1H), 6.23 (s, 1H), 1.38 (s, 9H). ${ }^{13} \mathrm{C}$ NMR (100 MHz, $\left.\mathrm{CDCl}_{3}\right) \delta$ 187.5, 185.4, 163.0 (d, $J=1.8 \mathrm{~Hz}$ ), 159.3 (d, $J=245.7 \mathrm{~Hz}), 148.8$ (d, $J=2.4 \mathrm{~Hz}), 137.1,137.0,136.2(\mathrm{~d}, J=3.5 \mathrm{~Hz}), 131.9$ (d, $J=9.7$ $\mathrm{Hz}), 131.4,126.4$ (d, $J=2.9 \mathrm{~Hz}), 124.7$ (d, $J=15.8 \mathrm{~Hz}$ ), 117.7 (d, $J$ $=24.5 \mathrm{~Hz}), 52.4,28.8$. HRMS: $[\mathrm{M}+\mathrm{H}]^{+}$calculated for $\mathrm{C}_{17} \mathrm{H}_{17} \mathrm{FNO}_{3}$ : 302.1192, found 302.1194.

$N$-(tert-butyl)-3-(3,6-dioxocyclohexa-1,4-dien-1-yl)-2naphthamide (3p). ${ }^{1} \mathrm{H}$ NMR (400 $\left.\mathrm{MHz}, \mathrm{CDCl}_{3}\right) \delta 7.97(\mathrm{~s}, 1 \mathrm{H})$, $7.87(\mathrm{dd}, J=11.5,4.7 \mathrm{~Hz}, 2 \mathrm{H}), 7.78(\mathrm{~s}, 1 \mathrm{H}), 7.62-7.55(\mathrm{~m}, 2 \mathrm{H})$, $6.86(\mathrm{~s}, 1 \mathrm{H}), 6.81(\mathrm{~s}, 2 \mathrm{H}), 6.18(\mathrm{~s}, 1 \mathrm{H}), 1.43$ (s, 9H). ${ }^{13} \mathrm{C}$ NMR (100 MHz, $\left.\mathrm{CDCl}_{3}\right) \delta 187.8,186.4,168.7,149.7,137.1,137.0$, 135.1, 133.9, 133.1, 131.2, 130.8, 130.4, 128.4, 128.3, 128.2, 128.1, 126.5, 52.1, 28.8. HRMS: $[\mathrm{M}+\mathrm{H}]^{+}$calculated for $\mathrm{C}_{21} \mathrm{H}_{20} \mathrm{NO}_{3}: 334.1443$, found 334.1442.

$N$-(tert-butyl)-3-(3,6-dioxocyclohexa-1,4-dien-1-yl)thiophene2-carboxamide (3q). ${ }^{1} \mathrm{H}$ NMR $\left(400 \mathrm{MHz}, \mathrm{CDCl}_{3}\right) \delta 7.35(\mathrm{~d}, J=5.0$ $\mathrm{Hz}, 1 \mathrm{H}), 6.99$ (d, $J=5.0 \mathrm{~Hz}, 1 \mathrm{H}), 6.86$ (d, $J=10.1 \mathrm{~Hz}, 1 \mathrm{H}), 6.80$ (dd, $J=10.1,2.3 \mathrm{~Hz}, 1 \mathrm{H}), 6.75$ (d, $J=2.3 \mathrm{~Hz}, 1 \mathrm{H}), 5.79(\mathrm{~s}, 1 \mathrm{H}$ ), 1.39 (s, 9H). ${ }^{13} \mathrm{C}$ NMR $\left(100 \mathrm{MHz}, \mathrm{CDCl}_{3}\right) \delta 187.4,185.0,161.4$, 143.6, 137.6, 137.2, 136.6, 134.9, 132.1, 129.7, 126.5, 52.4, 28.8. HRMS: $[\mathrm{M}+\mathrm{H}]^{+}$calculated for $\mathrm{C}_{15} \mathrm{H}_{16} \mathrm{NO}_{3} \mathrm{~S}$ : 290.0851 , found 290.0849 .

$N$-(tert-butyl)-2-(1,4-dioxo-1,4-dihydronaphthalen-2-yl) benzamide (3r). ${ }^{1} \mathrm{H}$ NMR (400 $\left.\mathrm{MHz}^{\mathrm{CDCl}}\right)_{3} \delta 8.14-8.08(\mathrm{~m}$, $1 \mathrm{H}), 8.06-8.00(\mathrm{~m}, 1 \mathrm{H}), 7.77-7.69(\mathrm{~m}, 2 \mathrm{H}), 7.58-7.52(\mathrm{~m}, 1 \mathrm{H})$, $7.52-7.43(\mathrm{~m}, 2 \mathrm{H}), 7.37(\mathrm{dd}, J=7.4,1.3 \mathrm{~Hz}, 1 \mathrm{H}), 6.98(\mathrm{~s}, 1 \mathrm{H})$, $6.03(\mathrm{~s}, 1 \mathrm{H}), 1.36(\mathrm{~s}, 9 \mathrm{H}) .{ }^{13} \mathrm{C} \mathrm{NMR}\left(100 \mathrm{MHz}, \mathrm{CDCl}_{3}\right) \delta 185.2$, 184.0, 168.7, 151.4, 138.1, 133.8, 133.7, 133.0, 132.7, 130.8, 130.5, 129.8, 126.8, 126.5, 126.3, 52.0, 28.8. Two carbons are not visible due to overlapping peaks. HRMS: $[\mathrm{M}+\mathrm{H}]^{+}$calculated for $\mathrm{C}_{21} \mathrm{H}_{20} \mathrm{NO}_{3}$ : 334.1443, found 334.1440.

$N$-(tert-butyl)-3',4'-dimethyl-2',5'-dioxo-2',5'-dihydro-[1,1'biphenyl]-2-carboxamide (3s). ${ }^{1} \mathrm{H}$ NMR (400 $\left.\mathrm{MHz} \mathrm{CDCl}_{3}\right) \delta$ 7.53-7.40 (m, 3H), 7.31-7.27 (m, 1H), $6.71(\mathrm{~s}, 1 \mathrm{H}), 5.98(\mathrm{~s}, 1 \mathrm{H})$, $2.06(\mathrm{~s}, 3 \mathrm{H}), 2.04(\mathrm{~s}, 3 \mathrm{H}), 1.39$ (s, 9H). ${ }^{13} \mathrm{C}$ NMR $(100 \mathrm{MHz}$, $\left.\mathrm{CDCl}_{3}\right) \delta 187.5,186.2,168.7,149.0,141.5,141.3,137.8,133.7$, 131.1, 130.8, 130.4, 129.6, 126.5, 52.0, 28.8, 12.7, 12.4. HRMS: $[\mathrm{M}+\mathrm{H}]^{+}$calculated for $\mathrm{C}_{19} \mathrm{H}_{22} \mathrm{NO}_{3}: 312.1600$, found 312.1605.

$N$-(tert-butyl)-4'-methyl-2',5'-dioxo-2',5'-dihydro-[1,1'biphenyl]-2-carboxamide and $N$-(tert-butyl)-3'-methyl-2',5'dioxo-2',5'-dihydro-[1,1'-biphenyl]-2-carboxamide (3t). Selected signals: Major: ${ }^{1} \mathrm{H} \mathrm{NMR}\left(400 \mathrm{MHz}, \mathrm{CDCl}_{3}\right) \delta 7.54-7.41(\mathrm{~m}$, 3H), 7.30-7.26 (m, 1H), $6.01(\mathrm{~s}, 1 \mathrm{H}), 2.06(\mathrm{~d}, J=1.5 \mathrm{~Hz}, 3 \mathrm{H})$, 1.39 (s, 9H). $\left.{ }^{13} \mathrm{C} \mathrm{NMR} \mathrm{(100} \mathrm{MHz,} \mathrm{CDCl}_{3}\right) \delta 187.6,186.5,168.6$, 149.6, 146.4, 137.6, 133.7, 131.3, 130.9, 130.4, 129.7, 126.5, 52.0, 28.7, 16.2. Minor: ${ }^{1} \mathrm{H}$ NMR (400 MHz, $\left.\mathrm{CDCl}_{3}\right) \delta 7.54-7.41$ $(\mathrm{m}, 3 \mathrm{H}), 7.30-7.26(\mathrm{~m}, 1 \mathrm{H}), 6.00(\mathrm{~s}, 1 \mathrm{H}), 2.09(\mathrm{~d}, J=1.5 \mathrm{~Hz}, 3 \mathrm{H})$, 1.39 (s, 9H). ${ }^{13} \mathrm{C}$ NMR $\left(100 \mathrm{MHz} \mathrm{CDCl}_{3}\right) \delta 188.1,186.1,168.6$, 149.3, 146.4, 137.8, 133.1, 131.5, 130.8, 130.5, 126.5, 52.0, 28.7, 15.8. HRMS: $[\mathrm{M}+\mathrm{H}]+$ calculated for $\mathrm{C}_{18} \mathrm{H}_{20} \mathrm{NO}_{3}: 298.1443$, found 
298.1447.

$N$-(tert-butyl)-4'-methoxy-2',5'-dioxo-2',5'-dihydro-[1,1'biphenyl]-2-carboxamide and $N$-(tert-butyl)-3'-methoxy-2',5'dioxo-2',5'-dihydro-[1,1'-biphenyl]-2-carboxamide (3u). Selected signals: Major: ${ }^{1} \mathrm{H}$ NMR $\left(400 \mathrm{MHz}, \mathrm{CDCl}_{3}\right) \delta 7.55-7.41(\mathrm{~m}$, $3 \mathrm{H}), 7.28(\mathrm{t}, J=6.2 \mathrm{~Hz}, 1 \mathrm{H}), 6.70(\mathrm{~s}, 1 \mathrm{H}), 6.05(\mathrm{~s}, 1 \mathrm{H}), 5.97(\mathrm{~s}$, $1 \mathrm{H}), 3.84(\mathrm{~s}, 3 \mathrm{H}), 1.40(\mathrm{~s}, 9 \mathrm{H}) .{ }^{13} \mathrm{C}$ NMR $\left(100 \mathrm{MHz}, \mathrm{CDCl}_{3}\right) \delta$ 185.8, 182.1, 168.4, 159.1, 150.0, 137.9, 133.1, 130.7, 130.4, $129.8,129.4,126.5,107.8,56.3,51.9,28.7$. Minor: ${ }^{1} \mathrm{H}$ NMR (400 $\left.\mathrm{MHz} \mathrm{CDCl}_{3}\right) \delta 7.55-7.41(\mathrm{~m}, 3 \mathrm{H}), 7.28(\mathrm{t}, J=6.2 \mathrm{~Hz}, 1 \mathrm{H}), 6.65(\mathrm{~d}$, $J=2.3 \mathrm{~Hz}, 1 \mathrm{H}), 6.05(\mathrm{~s}, 1 \mathrm{H}), 5.96(\mathrm{~d}, J=2.3 \mathrm{~Hz}, 1 \mathrm{H}), 3.82(\mathrm{~s}, 3 \mathrm{H})$, $1.38(\mathrm{~s}, 9 \mathrm{H}) .{ }^{13} \mathrm{C} \mathrm{NMR}\left(100 \mathrm{MHz}, \mathrm{CDCl}_{3}\right) \delta 187.3,180.6,171.2$, 159.4, 147.5, 137.4, 131.9, 130.8, 130.4, 129.7, 126.5, 107.7, 56.4, 52.0, 28.7. HRMS: $[\mathrm{M}+\mathrm{H}]^{+}$calculated for $\mathrm{C}_{18} \mathrm{H}_{20} \mathrm{NO}_{4}$ : 314.1392, found 314.1395.

$N$-(tert-butyl)-4'-chloro-2',5'-dioxo-2',5'-dihydro-[1,1'biphenyl]-2-carboxamide and $N$-(tert-butyl)-3'-chloro-2',5'dioxo-2',5'-dihydro-[1,1'-biphenyl]-2-carboxamide (3v). Selected ${ }^{1} \mathrm{H}$ NMR signals: 1) ${ }^{1} \mathrm{H}$ NMR (400 $\left.\mathrm{MHz}, \mathrm{CDCl}_{3}\right) \delta$ 7.55-7.45 (m, 3H), 7.32-7.27 (m, 1H), 6.05 (s, 1H), 1.39 (s, 9H). 2) ${ }^{1} \mathrm{H}$ NMR (400 MHz, $\left.\mathrm{CDCl}_{3}\right) \delta 7.55-7.45(\mathrm{~m}, 3 \mathrm{H}), 7.32-7.27$ (m, 1H), 6.05 (s, 1H), 1.39 (s, 9H). $\left.{ }^{13} \mathrm{C} \mathrm{NMR} \mathrm{(100} \mathrm{MHz,} \mathrm{CDCl}_{3}\right) \delta$ $185.10,183.62,179.75,178.53,168.40,168.28,150.29,149.84$, $144.64,144.45,137.68,137.50,134.20,134.07,133.15,132.68$, $131.08,131.00,130.54,130.33,130.20,126.49,126.47,52.28$, 52.15, 28.77, 28.76. HRMS: $[\mathrm{M}+\mathrm{H}]^{+}$calculated for $\mathrm{C}_{17} \mathrm{H}_{17} \mathrm{ClNO}_{3}$ : 318.0897, found 318.0903 .

$N$-(tert-butyl)-2',5'-dioxo-2',5'-dihydro-[1,1':4',1"-terphenyl]2-carboxamide and $N$-(tert-butyl)-2',5'-dioxo-2',5'-dihydro[1,1':3',1"-terphenyl]-2-carboxamide (3w). Selected signals: Major: ${ }^{1} \mathrm{H}$ NMR (400 MHz, $\left.\mathrm{CDCl}_{3}\right) \delta 6.85(\mathrm{~d}, J=2.6 \mathrm{~Hz}, 1 \mathrm{H}), 6.75$ $(\mathrm{d}, J=2.6 \mathrm{~Hz}, 1 \mathrm{H}), 6.11(\mathrm{~s}, 1 \mathrm{H}), 1.32(\mathrm{~s}, 9 \mathrm{H}) .{ }^{13} \mathrm{C}$ NMR $(100 \mathrm{MHz}$, $\left.\mathrm{CDCl}_{3}\right) \delta 187.6,185.4,168.1,150.2,146.8,137.1,133.9,133.4$, 132.8, 131.0, 130.9, 130.7, 129.8, 129.7, 129.4, 128.2, 126.6, 51.9, 28.7. Minor: ${ }^{1} \mathrm{H}$ NMR ( $\left.400 \mathrm{MHz}, \mathrm{CDCl}_{3}\right) \delta 6.89$ (s, 1H), 6.82 $(\mathrm{s}, 1 \mathrm{H}), 6.09$ (s, 1H), 1.39 (s, 9H). ${ }^{13} \mathrm{C} \mathrm{NMR}\left(100 \mathrm{MHz}, \mathrm{CDCl}_{3}\right) \delta$ $186.9,186.0,168.5,148.9,146.2,137.8,133.1,132.9,132.7$, 131.8, 130.5, 130.0, 129.5, 128.5, 126.52 51.9, 28.7. HRMS: [M + $\mathrm{H}]^{+}$calculated for $\mathrm{C}_{23} \mathrm{H}_{22} \mathrm{NO}_{3}: 360.1600$, found 360.1600 .

$\mathrm{N}$-(tert-butyl)-2-(3,6-dioxocyclohexa-1,4-dien-1-yl)cyclopent1-enecarboxamide (3x). ${ }^{1} \mathrm{H}$ NMR (400 MHz, $\left.\mathrm{CDCl}_{3}\right) \delta 6.82$ (d, $J=$ $10.2 \mathrm{~Hz}, 1 \mathrm{H}$ ), 6.73 (dd, $J=10.2,2.5 \mathrm{~Hz}, 1 \mathrm{H}$ ), 6.55 (d, $J=2.5 \mathrm{~Hz}$, $1 \mathrm{H}), 5.36(\mathrm{~s}, 1 \mathrm{H}), 2.76-2.66(\mathrm{~m}, 4 \mathrm{H}), 2.11-2.02(\mathrm{~m}, 2 \mathrm{H}), 1.31(\mathrm{~s}$, 9H). ${ }^{13} \mathrm{C}$ NMR $\left(100 \mathrm{MHz}, \mathrm{CDCl}_{3}\right) \delta 187.4,185.0,164.8,146.4$, 140.5, 139.9, 137.6, 136.5, 130.7, 51.6, 38.2, 34.9, 28.9, 22.6. HRMS: $[\mathrm{M}+\mathrm{H}]^{+}$calculated for $\mathrm{C}_{16} \mathrm{H}_{20} \mathrm{NO}_{3}$ : 274.1443, found 274.1446.

$N^{2}, N^{2}$ '-di-tert-butyl-2',5'-dioxo-2',5'-dihydro-[1,1':3',1'terphenyl]-2,2"-dicarboxamide (3aa). ${ }^{1} \mathrm{H}$ NMR $(400 \mathrm{MHz}$, $\left.\mathrm{CDCl}_{3}\right) \delta$ 7.56-7.52 (m, 2H), 7.49-7.40 (m, 6H), $6.80(\mathrm{~s}, 2 \mathrm{H})$, $6.04(\mathrm{~s}, 2 \mathrm{H}), 1.21(\mathrm{~s}, 9 \mathrm{H}) .{ }^{13} \mathrm{C} \mathrm{NMR}\left(100 \mathrm{MHz}, \mathrm{CDCl}_{3}\right) \delta 187.2$, 185.4, 168.4, 148.7, 137.8, 132.3, 132.2, 130.4, 130.2, 129.6, 127.3, 52.1, 28.7. HRMS: $[\mathrm{M}+\mathrm{H}]^{+}$calculated for $\mathrm{C}_{28} \mathrm{H}_{31} \mathrm{~N}_{2} \mathrm{O}_{4}$ : 459.2284, found 459.2288 .

$N$-isopropyl-2',5'-dioxo-2',5'-dihydro-[1,1'-biphenyl]-2carboxamide (3ba). ${ }^{1} \mathrm{H}$ NMR $\left(400 \mathrm{MHz}, \mathrm{CDCl}_{3}\right) \delta 7.57-7.43(\mathrm{~m}$, $3 \mathrm{H}), 7.30(\mathrm{~d}, J=7.2 \mathrm{~Hz}, 1 \mathrm{H}), 6.84-6.77(\mathrm{~m}, 2 \mathrm{H}), 6.74(\mathrm{~d}, J=1.8$
$\mathrm{Hz}, 1 \mathrm{H}), 6.02$ (d, $J=7.0 \mathrm{~Hz}, 1 \mathrm{H}), 4.12-4.00(\mathrm{~m}, 1 \mathrm{H}), 1.21(\mathrm{~d}, J=$ $6.6 \mathrm{~Hz}, 6 \mathrm{H}) .{ }^{13} \mathrm{C}$ NMR (100 MHz, $\left.\mathrm{CDCl}_{3}\right) \delta 187.6,185.9,168.0$, 149.4, 137.1, 136.9, 136.5, 133.4, 131.5, 131.1, 130.5, 129.9, 126.6, 42.3, 22.8. HRMS: $[\mathrm{M}+\mathrm{H}]^{+}$calculated for $\mathrm{C}_{16} \mathrm{H}_{16} \mathrm{NO}_{3}$ : 270.1130 , found 270.1134 .

$N$-cyclohexyl-2',5'-dioxo-2',5'-dihydro-[1,1'-biphenyl]-2carboxamide (3ca). ${ }^{1} \mathrm{H}$ NMR $\left(400 \mathrm{MHz}, \mathrm{CDCl}_{3}\right) \delta 7.58-7.45(\mathrm{~m}$, $3 \mathrm{H}), 7.30(\mathrm{~d}, J=7.5 \mathrm{~Hz}, 1 \mathrm{H}), 6.86-6.78(\mathrm{~m}, 2 \mathrm{H}), 6.74(\mathrm{~d}, J=2.0$ $\mathrm{Hz}, 1 \mathrm{H}), 5.99$ (d, $J=7.5 \mathrm{~Hz}, 1 \mathrm{H}), 3.82-3.71(\mathrm{~m}, 1 \mathrm{H}), 2.01-1.91$ (m, 2H), 1.79-1.69 (m, 2H), 1.63 (d, $J=10.6 \mathrm{~Hz}, 2 \mathrm{H}), 1.45-1.31$ $(\mathrm{m}, 2 \mathrm{H}), 1.27-1.16(\mathrm{~m}, 2 \mathrm{H}) .{ }^{13} \mathrm{C} \mathrm{NMR}\left(100 \mathrm{MHz}, \mathrm{CDCl}_{3}\right) \delta 187.6$, $185.9,167.9,149.5,137.2,136.9,136.6,133.4,131.5,131.1$, 130.5, 129.9, 126.6, 49.1, 33.2, 25.7, 25.0. HRMS: $[\mathrm{M}+\mathrm{H}]^{+}$calculated for $\mathrm{C}_{19} \mathrm{H}_{20} \mathrm{NO}_{3}$ : 310.1443, found 310.1445.

$N$-(1-adamantanyl)-2',5'-dioxo-2',5'-dihydro-[1,1'-biphenyl]2-carboxamide (3da). ${ }^{1} \mathrm{H}$ NMR $\left(400 \mathrm{MHz}^{\left.-\mathrm{CDCl}_{3}\right)} \delta\right.$ 7.56-7.42 (m, 3H), 7.31-7.26 (m, 1H), 6.86-6.78 (m, 2H), $6.74(\mathrm{~d}, J=2.0$ $\mathrm{Hz}, 1 \mathrm{H}), 5.84$ (s, 1H), 2.09 (s, 3H), 2.03 (d, $J=2.6 \mathrm{~Hz}, 6 \mathrm{H}), 1.69$ $(\mathrm{s}, 6 \mathrm{H}) .{ }^{13} \mathrm{C}$ NMR $\left(100 \mathrm{MHz}, \mathrm{CDCl}_{3}\right) \delta 187.6,185.8,168.2,149.6$, 137.8, 137.1, 137.1, 133.2, 131.3, 130.9, 130.5, 129.9, 126.5, 52.8, 41.6, 36.5, 29.6. HRMS: $[\mathrm{M}+\mathrm{H}]^{+}$calculated for $\mathrm{C}_{23} \mathrm{H}_{24} \mathrm{NO}_{3}$ : 362.1756, found 362.1761 .

2-Hydroxy-6H-benzo[c]chromen-6-one (4a). ${ }^{1} \mathrm{H}$ NMR (400 MHz, DMSO-d6) $\delta 9.73(\mathrm{~s}, 1 \mathrm{H}), 8.33-8.21(\mathrm{~m}, 2 \mathrm{H}), 7.98-7.91(\mathrm{~m}$, 1H), 7.72-7.66 (m, 1H), $7.62(\mathrm{~d}, J=2.7 \mathrm{~Hz}, 1 \mathrm{H}), 7.28(\mathrm{~d}, J=8.9$ $\mathrm{Hz}, 1 \mathrm{H}), 7.02$ (dd, $J=8.9,2.8 \mathrm{~Hz}, 1 \mathrm{H}) .{ }^{13} \mathrm{C} \mathrm{NMR}(100 \mathrm{MHz}$, DMSO-d6) $\delta 160.9,154.7,144.4,135.7,134.7,130.2,129.7$, 122.9, 121.1, 118.9, 118.7, 118.6, 108.7. HRMS: [M + H]+ calculated for $\mathrm{C}_{13} \mathrm{H}_{9} \mathrm{O}_{3}: 213.0555$, found 213.0552.

2-Hydroxy-8-methyl-6H-benzo[c]chromen-6-one (4b). ${ }^{1} \mathrm{H}$ NMR (400 MHz, DMSO-d $) \delta 9.69(\mathrm{~s}, 1 \mathrm{H}), 8.15(\mathrm{~d}, J=8.1 \mathrm{~Hz}$, $1 \mathrm{H}), 8.04(\mathrm{~s}, 1 \mathrm{H}), 7.74(\mathrm{~d}, J=8.1 \mathrm{~Hz}, 1 \mathrm{H}), 7.56(\mathrm{~d}, J=2.3 \mathrm{~Hz}, 1 \mathrm{H})$, $7.25(\mathrm{~d}, J=8.8 \mathrm{~Hz}, 1 \mathrm{H}), 6.97(\mathrm{dd}, J=8.8,2.5 \mathrm{~Hz}, 1 \mathrm{H}), 2.46(\mathrm{~s}, 3 \mathrm{H})$. ${ }^{13} \mathrm{C}$ NMR (100 MHz, DMSO- $\left.d_{6}\right) \delta 160.9,154.7,144.1,139.6$, 136.7, 132.2, 129.9, 122.9, 120.9, 118.8, 118.46, 118.4, 108.4, 21.2. HRMS: $[\mathrm{M}+\mathrm{H}]^{+}$calculated for $\mathrm{C}_{14} \mathrm{H}_{11} \mathrm{O}_{3}$ : 227.0708 , found 227.0706.

9-Chloro-2-hydroxy-6H-benzo[c]chromen-6-one (4c). ${ }^{1} \mathrm{H}$ NMR (400 MHz, DMSO-d6) $\delta 9.74(\mathrm{~s}, 1 \mathrm{H}), 8.38(\mathrm{~d}, J=1.9 \mathrm{~Hz}$, $1 \mathrm{H}), 8.22(\mathrm{~d}, J=8.5 \mathrm{~Hz}, 1 \mathrm{H}), 7.70(\mathrm{dd}, J=8.5,1.9 \mathrm{~Hz}, 1 \mathrm{H}), 7.65$ (d, $J=2.7 \mathrm{~Hz}, 1 \mathrm{H}$ ), 7.27 (d, $J=8.9 \mathrm{~Hz}, 1 \mathrm{H}$ ), 7.04 (dd, $J=8.9,2.7$ Hz, 1H). ${ }^{13} \mathrm{C}$ NMR (100 MHz, DMSO- $\left.d 6\right) \delta 160.2,154.8,144.8$, 141.1, 136.6, 132.4, 129.8, 122.89, 119.9, 119.6, 118.6, 117.8, 109.2. HRMS: $[\mathrm{M}+\mathrm{H}]^{+}$calculated for $\mathrm{C}_{13} \mathrm{H}_{8} \mathrm{ClO}_{3}$ : 247.0162, found 247.0161.

9-Bromo-2-hydroxy-6H-benzo[c]chromen-6-one (4d). ${ }^{1} \mathrm{H}$ NMR (400 MHz, DMSO- $\left.d_{6}\right) \delta 9.73(\mathrm{~d}, J=3.8 \mathrm{~Hz}, 1 \mathrm{H}), 8.48(\mathrm{~d}, J=$ $11.4 \mathrm{~Hz}, 1 \mathrm{H}), 8.12(\mathrm{t}, J=8.0 \mathrm{~Hz}, 1 \mathrm{H}), 7.82(\mathrm{t}, J=7.9 \mathrm{~Hz}, 1 \mathrm{H})$, 7.69-7.59 (m, 1H), $7.26(\mathrm{dd}, J=8.6,6.9 \mathrm{~Hz}, 1 \mathrm{H}), 7.04(\mathrm{dt}, J=8.8$, $3.2 \mathrm{~Hz}, 1 \mathrm{H}) .{ }^{13} \mathrm{C}$ NMR $\left(100 \mathrm{MHz}\right.$, DMSO- $\left.d_{6}\right) \delta 160.4,154.8,144.7$, 136.6, 132.6, 132.2, 130.3, 125.8, 120.2, 119.6, 118.6, 117.6, 109.2. HRMS: $[\mathrm{M}+\mathrm{H}]^{+}$calculated for $\mathrm{C}_{13} \mathrm{H}_{8} \mathrm{BrO}_{3}$ : 290.9657, found 290.9659.

\section{Results and discussion}

The reaction of $N$-(tert-butyl)benzamide $\mathbf{1 a}$ and 1,4-benzo- 
quinone 2a was selected as a model for screening of the reaction parameters (Table 1). We initially performed this reaction in the absence of any external oxidant but with $4 \mathrm{~mol} \%$ of [RhCp* $\left.\mathrm{Cl}_{2}\right]_{2}$ and $16 \mathrm{~mol} \%$ of $\mathrm{AgSbF}_{6}$. Only traces of the desired product 3a were detected by GC-MS (Table 1, entry 1). When $\mathrm{Cu}(\mathrm{OAc})_{2}$ (1 equiv) was employed as an oxidant, 3a was isolated in $17 \%$ yield (entry 2). However, no product was obtained when $\mathrm{AgSbF}_{6}$ was omitted (entry 3). With $\mathrm{Ag}_{2} \mathrm{CO}_{3}$ or $\mathrm{AgOAc}$ being an oxidant, the yield of 3a was increased to $54 \%$ and $21 \%$, respectively (entries 4 and 5). We were pleased to find that $3 \mathbf{a}$ was isolated in $85 \%$ yield when the amount of $\mathrm{Ag}_{2} \mathrm{CO}_{3}$ was increased to 2.0 equiv (entry 6). Further examination of the solvent revealed that DCE, THF, diglyme, $\mathrm{PhCl}$, and acetone all gave inferior results (entries 7-11). Finally, the optimized conditions include $4 \mathrm{~mol} \%$ of $\left[\mathrm{RhCp}^{*} \mathrm{Cl}_{2}\right]_{2}$ as catalyst, $16 \mathrm{~mol} \%$ of $\mathrm{AgSbF}_{6}$ as an additive, and 2.0 equiv of $\mathrm{Ag}_{2} \mathrm{CO}_{3}$ in 1,4-dioxane at $100{ }^{\circ} \mathrm{C}$.

Having determined the optimal conditions, we next surveyed various substrates to define the scope of the reaction (Table 2). $\mathrm{N}$-(tert-butyl)benzamides bearing electron-donating groups at the para-position gave the corresponding products in moderate to good yields (3a-3c, 3g). With a halogen group at the 4-position of phenyl ring, the arylated quinones were isolated in slightly lower yield (3d-3f). However, using electron-withdrawing groups such as trifluoromethyl and methoxycarbonyl at the para-position decreased the yield of the products ( $\mathbf{3 h}$ and $\mathbf{3 i}$ ). The meta methyl substituted substrate also gave product in good yield $(\mathbf{3} \mathbf{j})$ and $\mathrm{C}-\mathrm{H}$ activation occurred selectively at the less hindered position. However, the meta methoxy (3k and $\mathbf{3} \mathbf{k}^{\prime}$ ) or chloro (3l and $\mathbf{3} \mathbf{l}^{\prime}$ ) substituted benzamide gave two regio-isomeric products that could be chromatographically separated. In contrast, $\mathrm{C}-\mathrm{H}$ activation occurred selectively at the more hindered ortho site for the meta fluoro substituted benzamide $(\mathbf{3 m})$, on the basis of ${ }^{1} \mathrm{H}$ and ${ }^{13} \mathrm{C}$ NMR analysis. ortho-Methoxy substituted benzamide was also effective, furnishing the arylated quinone in $68 \%$ yield (3n).

\section{Table 1}

Coupling of $N$-(tert-butyl)benzamide with quinone under various conditions.

\begin{tabular}{|c|c|c|c|c|}
\hline & $\mathrm{HN}^{-\mathrm{t}-\mathrm{Bu}}$ & $\underset{\text { solvent, } 100^{\circ} \mathrm{C}, \mathrm{N}_{2}}{\stackrel{\text { catalyst, oxidant }}{\longrightarrow}}$ & & \\
\hline Entry & Catalyst & Oxidant (equiv) & Solvent & $\begin{array}{r}\text { Isolated } \\
\text { yield (\%) }\end{array}$ \\
\hline 1 & {$\left[\mathrm{RhCp}^{*} \mathrm{Cl}_{2}\right]_{2} / \mathrm{AgSbF}_{6}$} & - & Dioxane & Trace \\
\hline 2 & {$\left[\mathrm{RhCp}^{*} \mathrm{Cl}_{2}\right]_{2} / \mathrm{AgSbF}_{6}$} & $\mathrm{Cu}(\mathrm{OAc})_{2}(1.0)$ & Dioxane & 17 \\
\hline 3 & {$\left[\mathrm{RhCp}^{*} \mathrm{Cl}_{2}\right]_{2}$} & $\mathrm{Cu}(\mathrm{OAc})_{2}(1.0)$ & Dioxane & nd \\
\hline 4 & {$\left[\mathrm{RhCp}^{*} \mathrm{Cl}_{2}\right]_{2} / \mathrm{AgSbF}_{6}$} & $\mathrm{Ag}_{2} \mathrm{CO}_{3}(1.0)$ & Dioxane & 54 \\
\hline 5 & {$\left[\mathrm{RhCp}^{*} \mathrm{Cl}_{2}\right]_{2} / \mathrm{AgSbF}_{6}$} & AgOAc (1.0) & Dioxane & 21 \\
\hline 6 & {$\left[\mathrm{RhCp}^{*} \mathrm{Cl}_{2}\right]_{2} / \mathrm{AgSbF}_{6}$} & $\mathrm{Ag}_{2} \mathrm{CO}_{3}(2.0)$ & Dioxane & 85 \\
\hline 7 & {$\left[\mathrm{RhCp}^{*} \mathrm{Cl}_{2}\right]_{2} / \mathrm{AgSbF}_{6}$} & $\mathrm{Ag}_{2} \mathrm{CO}_{3}(2.0)$ & DCE & 73 \\
\hline 8 & {$\left[\mathrm{RhCp}^{*} \mathrm{Cl}_{2}\right]_{2} / \mathrm{AgSbF}_{6}$} & $\mathrm{Ag}_{2} \mathrm{CO}_{3}(2.0)$ & THF & 60 \\
\hline 9 & {$\left[\mathrm{RhCp}^{*} \mathrm{Cl}_{2}\right]_{2} / \mathrm{AgSbF}_{6}$} & $\mathrm{Ag}_{2} \mathrm{CO}_{3}(2.0)$ & Diglyme & Trace \\
\hline 10 & {$\left[\mathrm{RhCp}^{*} \mathrm{Cl}_{2}\right]_{2} / \mathrm{AgSbF}_{6}$} & $\mathrm{Ag}_{2} \mathrm{CO}_{3}(2.0)$ & $\mathrm{PhCl}$ & 36 \\
\hline 11 & {$\left[\mathrm{RhCp}^{*} \mathrm{Cl}_{2}\right]_{2} / \mathrm{AgSbF}_{6}$} & $\mathrm{Ag}_{2} \mathrm{CO}_{3}(2.0)$ & Acetone & 41 \\
\hline
\end{tabular}

Reactions conditions: $\left[\mathrm{RhCp}^{*} \mathrm{Cl}_{2}\right]_{2}$ (4 mol\%), $\mathrm{AgSbF}_{6}(16 \mathrm{~mol} \%)$, oxidant, $1 \mathbf{a}(0.20 \mathrm{mmol})$, and $2 \mathbf{a}(0.30 \mathrm{mmol})$ in solvent $(3 \mathrm{~mL})$ at $100{ }^{\circ} \mathrm{C}$ under nitrogen for $12 \mathrm{~h}$.
However, ortho-fluoro substrate exhibited lower reactivity (3o), implying that the electronic effect seems more significant than the steric effect at this position. Notably, the reactions for $N$-(tert-butyl)-2-naphthamide and $N$-(tert-butyl)thiophene-2carboxamide proceeded smoothly to generate products in moderate yields (3p and $\mathbf{3 q}$ ). The $N$-substituent is not limited to a ${ }^{\text {Bu }}$ group. Thus benzamides bearing $N$-alkyl groups such as isopropyl, cyclohexyl, and adamantyl also underwent smooth coupling (3ba-3da). The scope of quinone substrate was next investigated. Subjection of 1,4-naphthoquinone and 2,3-dimethyl-1,4-benzoquinone to the coupling with $\mathbf{1 a}$ afforded $\mathbf{3 r}$ and 3s in $90 \%$ and $64 \%$ yields, respectively. In contrast, the coupling of a mono-substituted quinone with 1a provided two regioisomers in moderate total yields $(\mathbf{3 t}-\mathbf{3 w})$. Furthermore, extension to an olefinic substrate bearing this type of directing group proved successful, where product $\mathbf{3 x}$ was isolated in $52 \%$ yield. In addition, when an excess (3.0 equiv) of 1 a was used, the diarylation product was isolated as the major one (3aa).

It is noteworthy that a $\mathrm{C}-\mathrm{H}$ insertion/lactonization product 4a was observed during the screening studies. It has been reported that the coupling of benzoic acid and benzoquinone afforded the same 2-hydroxy-6H-benzo[c]chromen-6-one under Ir(III) catalysis, however a rather high catalyst loading and a relatively high temperature were necessary to give synthetically useful yields [91]. Encouraged by these results, the reaction parameters were optimized for the synthesis of $\mathbf{4 a}$. It was found that benzamides bearing methyl (4b), chloro (4c), and bromo (4d) groups at the 3- or 4- position underwent smoothly coupling under these redox-neutral conditions in acetone to give lactones in moderate to good yields (Scheme 1). However, we attempted but failed to achieve this type reaction for 1,4-naphthoquinone, where only quinone $3 \mathbf{r}$ was isolated. We noted that lactonization via $\mathrm{C}-\mathrm{H}$ activation of other amides has been reported [92-94].

To probe the mechanism of this direct arylation of quinones, preliminary mechanistic studies were carried out. A notable primary kinetic isotope effect (KIE, $\left.k_{\mathrm{H}} / k_{\mathrm{D}}=4.6\right)$ was observed, indicating that $\mathrm{C}-\mathrm{H}$ bond cleavage of $\mathbf{1 a}$ is probably involved in the rate-limiting step. Furthermore, a competition reaction has been performed, where two benzamides $\mathbf{1 b}$ and $\mathbf{1 e}$ differing in electronic effects were allowed to compete in the coupling with 2a (1b:1e:2a $=1: 1: 1$ ratio). ${ }^{1} \mathrm{H}$ NMR analysis of the resulting mixture revealed that $\mathbf{3 b}$ and $\mathbf{3 e}$ were generated in 2.2:1 ratio, suggesting that coupling of an electron-rich arene is faster than that of a more electron-deficient one.

Based on these experiments and literature precedents $[58,95]$, a plausible mechanism is proposed in Scheme 2 . O-Coordination of the amide to the Rh(III) center and subsequent ortho $\mathrm{C}-\mathrm{H}$ bond activation afforded a five-membered rhodacyclic intermediate $\mathbf{A}$. Insertion of quinone $\mathbf{2 a}$ to $\mathrm{Rh}-\mathrm{C}$ bond of $\mathbf{A}$ gave a seven-membered rhodacycle $\mathbf{B}$, which is followed by epimerization at one of the $\alpha$-positions, allowing for $\beta$-hydrogen elimination to provide the desired product 3a together with the Rh(I) species (with elimination of HX). The Rh(I) was re-oxidized by $\mathrm{Ag}_{2} \mathrm{CO}_{3}$ to regenerate $\mathrm{Rh}$ (III) for the next catalytic cycle. 
Table 2

Substrate scope.

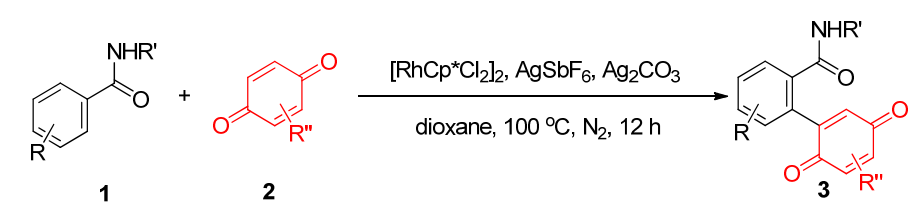

Entry

1

(a)

2<smiles>CCCCNC(=O)c1ccc(C)cc1C1=CC(=O)C=CC1=O</smiles>

3<smiles>CCCCCCCCNC(=O)c1ccc(OC)cc1C1=CC(=O)C=CC1=O</smiles>

4<smiles>CCCCCCNC(=O)C1C(=O)C=CC(=O)C1C1=CC(=O)C=CC1=O</smiles>

5<smiles>CCCCNC(=O)c1ccc(Cl)cc1C1=CC(=O)C=CC1=O</smiles>

6<smiles>CCCCNC(=O)c1ccc(Br)cc1C1=CC(=O)C=CC1=O</smiles>

7

8<smiles>CCCCCCNC(=O)c1ccc(-c2ccccc2)cc1C1=CC(=O)C=CC1=O</smiles><smiles>CCCCCCCNC(=O)C1=CC(=O)C=CC1=C1CC(=O)C=CC1=O</smiles>

9<smiles>CCCCCCCCCCNC(=O)c1ccc(C(C)=O)cc1C1=CC(=O)C=CC1=O</smiles>

10

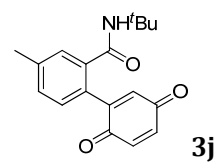

$\begin{gathered}\text { Isolated } \\ \text { yield (\%) }\end{gathered}$
Entry

66

15<smiles>CCCCNC(=O)c1cccc(F)c1C1=CC(=O)C=CC1=O</smiles>

67

68

68<smiles>CCCCCNC(=O)c1c(OC)cccc1C1=CC(=O)C=CC1=O</smiles>

17

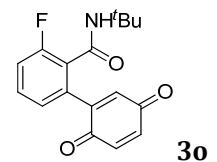

52

18<smiles>O=C1C=CC(=C2C(=O)C=CC(=O)C2=O)C=C1</smiles>

39

19

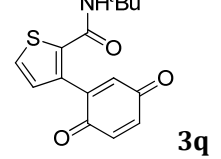

65

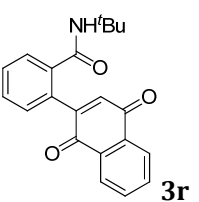

90
(1:1)

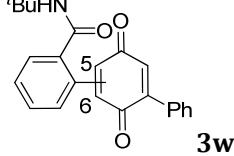

70

(major:minor $=6: 1$ )

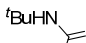

26

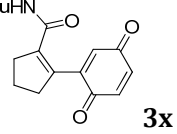

$27^{\text {a }}$<smiles>CCCCNC(=O)c1ccccc1C1=CC(=O)C=C(c2ccccc2C(N)=O)C1=O</smiles>

28
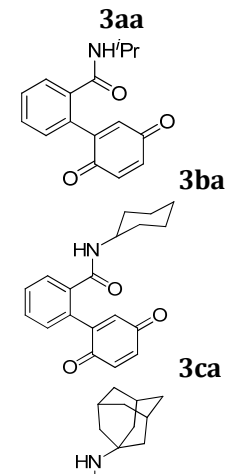

30

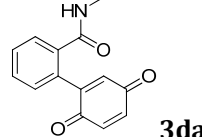




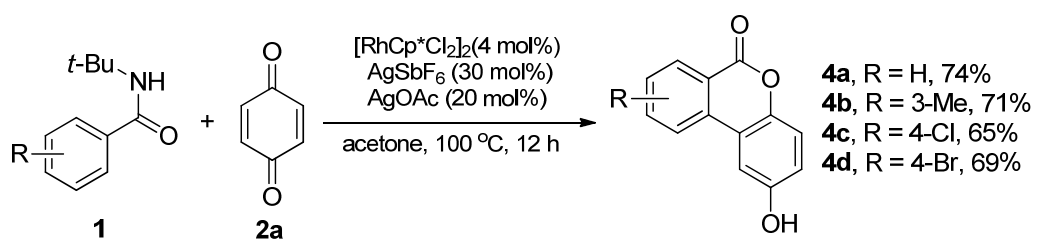

Scheme 1. Rh(III)-catalyzed synthesis of 2-hydroxy-6H-benzo[c]chromen-6-ones.

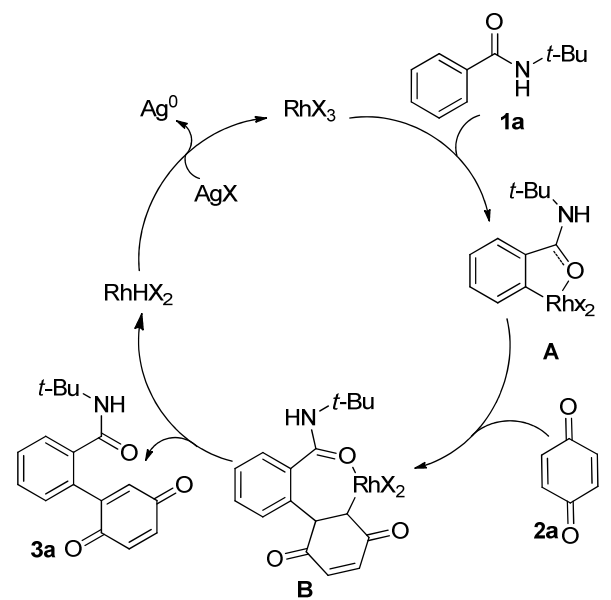

Scheme 2. Proposed mechanism of arylation of quinones.

\section{Conclusions}

We have developed $\mathrm{Rh}(\mathrm{III})$-catalyzed $\mathrm{C}-\mathrm{H}$ activation of $N$-(alkyl)benzamides in the coupling with quinones. A broad scope of substrates has been examined and good functional group compatibility has been realized. In addition 2-hydroxy-6H-benzo[c]chromen-6-ones were obtained via cascade reaction involving $\mathrm{C}-\mathrm{H}$ activation and arylation-lactonization reaction. Competition experiment and KIE study were conducted, and a plausible mechanism has been proposed. This method may find useful applications in the synthesis of complex structures.

\section{References}

[1] Gutekunst W R, Baran P S. Chem Soc Rev, 2011, 40: 1976

[2] Yamaguchi J, Yamaguchi A D, Itami K. Angew Chem Int Ed, 2012, 51: 8960

[3] McMurray L, O'Hara F, Gaunt M J. Chem Soc Rev, 2011, 40: 1885

[4] Daugulis O, Do H Q, Shabashov D. Acc Chem Res, 2009, 42: 1074

[5] Ackermann L, Vicente R, Kapdi A R. Angew Chem Int Ed, 2009, 48: 9792

[6] Lyons T W, Sanford M S. Chem Rev, 2010, 110: 1147

[7] Colby D A, Bergman R G, Ellman J A. Chem Rev, 2010, 110: 624

[8] Ackermann L. Chem Rev, 2011, 111: 1315

[9] Wencel-Delord J, Droge T, Liu F, Glorius F. Chem Soc Rev, 2011, 40: 4740

[10] Yeung C S, Dong V M. Chem Rev, 2011, 111: 1215

[11] Cho S H, Kim J Y, Kwak J, Chang S. Chem Soc Rev, 2011, 40: 5068

[12] Wendlandt A E, Suess A M, Stahl S S. Angew Chem Int Ed, 2011, 50: 11062

[13] Kuhl N, Hopkinson M N, Wencel-Delord J, Glorius F. Angew Chem Int Ed, 2012, 51: 10236
[14] Wu X F, Neumann H, Beller M. Chem Rev, 2012, 113: 1

[15] Engle K M, Mei T S, Wasa M, Yu J Q. Acc Chem Res, 2012, 45: 788

[16] Li D D, He C L, Cai H T, Wang G W. Chin J Org Chem (李丹丹, 何程 林, 蔡海婷, 王官武. 有机化学), 2013, 33: 203

[17] Yan G B, Wu X M, Yang M H. Org Biomol Chem, 2013, 11: 5558

[18] Ackermann L. Acc Chem Res, 2014, 47: 281

[19] Zhang M, Zhang Y F, Jie X M, Zhao H Q, Li G, Su W P. Org Chem Front, 2014, 1: 843

[20] Colby D A, Tsai A S, Bergman R G, Ellman J A. Acc Chem Res, 2011, 45: 814

[21] Song G Y, Wang F, Li X W. Chem Soc Rev, 2012, 41: 3651

[22] Kuhl N, Schröder N, Glorius F. Adv Synth Catal, 2014, 356: 1443

[23] Rakshit S, Grohmann C, Besset T, Glorius F. J Am Chem Soc, 2011, 133: 2350

[24] Huang X L, Huang J S, Du C L, Zhang X Y, Song F J, You J S. Angew Chem Int Ed, 2013, 52: 12970

[25] Liu B Q, Fan Y, Gao Y, Sun C, Xu C, Zhu J. J Am Chem Soc, 2013, 135: 468

[26] Neely J M, Rovis T. J Am Chem Soc, 2013, 135: 66

[27] Shen Y Y, Liu G X, Zhou Z, Lu X Y. Org Lett, 2013, 15: 3366

[28] Unoh Y, Hashimoto Y, Takeda D, Hirano K, Satoh T, Miura M. Org Lett, 2013, 15: 3258

[29] Zhang X S, Zhu Q L, Zhang Y F, Li Y B, Shi Z J. Chem Eur J, 2013, 19: 11898

[30] Dong Y, Liu G. Chem Commun, 2013, 49: 8066

[31] Li B, Ma J F, Xie W J, Song H B, Xu S S, Wang B Q. Chem Eur J, 2013, 19: 11863

[32] Zhao P, Wang F, Han K L, Li X W. Org Lett, 2012, 14: 3400

[33] Zhao P, Niu R, Wang F, Han K L, Li X W. Org Lett, 2012, 14: 4166

[34] Zhang T, Wu L M, Li X W. Org Lett, 2013, 15: 6294

[35] Wang F, Song G Y, Du Z Y, Li X W.J Org Chem, 2011, 76: 2926

[36] Wang F, Song G Y, Li X W. Org Lett, 2010, 12: 5430

[37] Umeda N, Hirano K, Satoh T, Miura M.J Org Chem, 2009, 74: 7094

[38] Guimond N, Gouliaras C, Fagnou K. J Am Chem Soc, 2010, 132: 6908

[39] Du Y, Hyster T K, Rovis T. Chem Commun, 2011, 47: 12074

[40] Hesp K D, Bergman R G, Ellman J A. J Am Chem Soc, 2011, 133: 11430

[41] Li Y, Li B J, Wang W H, Huang W P, Zhang X S, Chen K, Shi Z J. Angew Chem Int Ed, 2011, 50: 2115

[42] Tsai A S, Tauchert M E, Bergman R G, Ellman J A. J Am Chem Soc, 2011, 133: 1248

[43] Zhu C, Xie W Q, Falck J R. Chem Eur J, 2011, 17: 12591

[44] Chan W W, Lo S F, Zhou Z Y, Yu W Y. J Am Chem Soc, 2012, 134: 13565

[45] Karthikeyan J, Haridharan R, Cheng C H. Angew Chem Int Ed, 2012, 51: 12343

[46] Kim J Y, Park S H, Ryu J, Cho S H, Kim S H, Chang S. J Am Chem Soc, 2012, 134: 9110

[47] Schröder N, Wencel-Delord J, Glorius F. J Am Chem Soc, 2012, 134: 8298

[48] Wencel-Delord J, Nimphius C, Wang H G, Glorius F. Angew Chem 


\title{
Graphical Abstract
}

Chin. J. Catal., 2015, 36: 48-56 doi: 10.1016/S1872-2067(14)60245-X

\section{Rh(III)-catalyzed C-H activation of benzamides: Coupling with quinones}

Zisong Qi, Xingwei Li*

Dalian Institute of Chemical Physics, Chinese Academy of Sciences
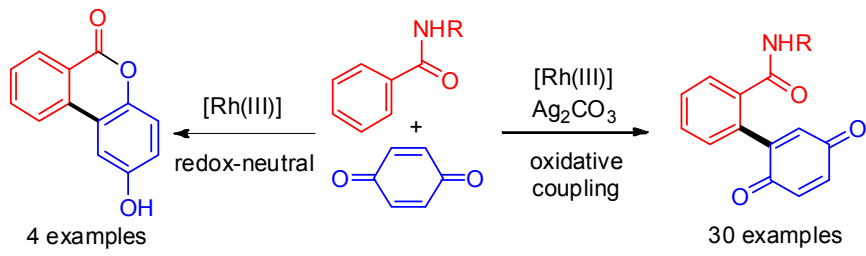

$\mathrm{Rh}(\mathrm{III})$-catalyzed C-H activation of $N$-(alkyl)benzamides in the oxidative coupling with various quinones. In addition, under redox-neutral conditions, 2-hydroxy- $6 H$-benzo[ $c]$ chromen-6-ones were also obtained via a cascade of cross-coupling followed by lactonization.

Int Ed, 2012, 51: 13001

[49] Cui S L, Zhang Y, Wu Q F. Chem Sci, 2013, 4: 3421

[50] Gong T J, Xiao B, Cheng W M, Su W, Xu J, Liu Z J, Liu L, Fu Y. J Am Chem Soc, 2013, 135: 10630

[51] Hyster T K, Ruhl K E, Rovis T. J Am Chem Soc, 2013, 135: 5364

[52] Li X W, Yu S J, Wang F, Wan B S, Yu X Z. Angew Chem Int Ed, 2013, 52: 2577

[53] Zhen W C, Du Z Y, Li X W. Chin J Catal (甄文萃, 杜正银, 李兴伟. 催 化学报), 2013, 34: 679

[54] Lian Y J, Hummel J R, Bergman R G, Ellman J A. J Am Chem Soc, 2013, 135: 12548

[55] Qi Z S, Li X W. Angew Chem Int Ed, 2013, 52: 8995

[56] Xie F, Qi Z S, Li X W. Angew Chem Int Ed, 2013, 52: 11862

[57] Zeng R, Wu S Z, Fu C L, Ma S M. J Am Chem Soc, 2013, 135: 18284

[58] Samanta R, Narayan R, Antonchick A P. Org Lett, 2012, 14: 6108

[59] Wang F, Song G Y, Du Z Y, Li X W. J Org Chem, 2011, 76: 2926

[60] Yang L, Correia C A, Li C J. Org Biomol Chem, 2011, 9: 7176

[61] Yang L, Qian B, Huang H M. Chem Eur J, 2012, 18: 9511

[62] Zhu C, Falck J R. Chem Commun, 2012, 48: 1674

[63] Zhang X Y, Wang F, Qi Z S, Yu S J, Li X W. Org Lett, 2014, 16: 1586

[64] Gould S J. Chem Rev, 1997, 97: 2499

[65] Marco-Contelles J, Molina M T. Curr Org Chem, 2003, 7: 1433

[66] Liu J K. Chem Rev, 2006, 106: 2209

[67] Zhang B, Salituro G, Szalkowski D, Li Z H, Zhang Y, Royo I, Vilella D, Díez M T, Pelaez F, Ruby C, Kendall R L, Mao X Z, Griffin P, Calaycay J, Zierath J R, Heck J V, Smith R G, Moller D E. Science, 1999, 284: 974

[68] Koyama J. Recent Pat Anti-Infect Drug Discovery, 2006, 1: 113

[69] Verma R P. Anti-Cancer Agents Med Chem, 2006, 6: 489

[70] Babula P, Adam V, Havel L, Kizek R. Ceska Slovens Farm, 2007, 56: 114

[71] Ferreira I C F R, Vaz J A, Vasconcelos M H, Martins A. Anti-Cancer Agents Med Chem, 2010, 10: 424

[72] Dandawate P R, Vyas A C, Padhye S B, Singh M W, Baruah J B. Mini-Rev Med Chem, 2010, 10: 436
[73] Nowicka B, Kruk J. Biochim Biophys Acta, 2010, 1797: 1587

[74] Cramer W A, Crofts A R. In: Photosynthesis. Vol. 1. New York: Academic Press, 1982. 387-467

[75] Bechtold T. In: Bechtold T, Mussak R, Eds. Handbook of Natural Colorants. New York: Wiley, 2009. 151-182

[76] Lin A J, Sartorelli A C. J Med Chem, 1976, 19: 1336

[77] Fujiwara Y, Domingo V, Seiple I B, Gianatassio R, Del Bel M, Baran P S. J Am Chem Soc, 2011, 133: 3292

[78] Wang J, Wang S, Wang G, Zhang J, Yu X Q. Chem commun, 2012, 48: 11769

[79] Deb A, Manna S, Maji A, Dutta U, Maiti D. Eur J Org Chem, 2013, 2013: 5251

[80] Echavarren A M, de Frutos Ó, Tamayo N, Noheda P, Calle P. J Org Chem, 1997, 62: 4524

[81] Tamayo N, Echavarren A M, Paredes M C. J Org Chem, 1991, 56: 6488

[82] Liebeskind L S, Riesinger S W. J Org Chem, 1993, 58: 408

[83] Gan X W, Jiang W, Wang W, Hu L H. Org Lett, 2009, 11: 589

[84] Itahara T. J Org Chem, 1985, 50: 5546

[85] Engler T A, Reddy J P.J Org Chem, 1991, 56: 6491

[86] Molina M T, Navarro C, Moreno A, Csákÿ A G. Org Lett, 2009, 11: 4938

[87] Demchuk O M, Pietrusiewicz K M. Syntlett, 2009: 1149

[88] Zhang S, Song F J, Zhao D B, You J S. Chem Commun, 2013, 49: 4558

[89] de Oliveira R A, Carazza F, da Silva Pereira M O. Synth Commun, 2000, 30: 4563

[90] Yoshida K, Oga N, Kadota M, Ogasahara Y, Kubo Y. J Chem Soc, Chem Commun, 1992: 1114

[91] Engelman K L, Feng Y, Ison E A. Organometallics, 2011, 30: 4572

[92] Zeng R, Fu C L, Ma S M. J Am Chem Soc, 2012, 134: 9597

[93] Wang F, Qi Z S, Sun J Q, Zhang X L, Li X W. Org Lett, 2013, 15: 6290

[94] Yang W, Sun J Q, Xu X X, Zhang Q, Liu Q. Chem Commun, 2014, 50: 4420

[95] Ryu J, Shin K, Park S H, Kim J Y, Chang S. Angew Chem Int Ed, 2012, 51: 9904

\section{三价铑催化苯甲酰胺与醌的 $\mathbf{C}-\mathbf{H}$ 活化偶联}

\author{
戚自松, 李兴伟* \\ 中国科学院大连化学物理研究所, 辽宁大连 116023
}


摘要: 三价铑在氧化条件下催化 $N$-烷基苯甲酰胺与醌反应生成芳基醌. 在氧化还原中性条件下, 经过偶联和内酯化反应可以得到 2 -拳基- $6 H$-苯并 $[c]$ 吡喃-6-酮.

关键词: 三价铑；碳氢活化；苯甲酰胺；芳基醌

收稿日期: 2014-09-25. 接受日期: 2014-11-10. 出版日期: 2015-01-20.

*通讯联系人. 电话: (0411)84379089; 电子信箱: xwli@dicp.ac.cn

基金来源: 中国科学院大连化学物理研究所; 国家自然科学基金(21272231).

本文的英文电子版由Elsevier出版社在ScienceDirect上出版(http://www.sciencedirect.com/science/journal/18722067). 